\title{
On the notions of absolute continuity for functions of several variables
}

\author{
by \\ Stanislav Hencl (Praha)
}

\begin{abstract}
Absolutely continuous functions of $n$ variables were recently introduced by J. Malý [5]. We introduce a more general definition, suggested by L. Zajíček. This new absolute continuity also implies continuity, weak differentiability with gradient in $L^{n}$, differentiability almost everywhere and the area formula. It is shown that our definition does not depend on the shape of balls in the definition.
\end{abstract}

1. Introduction. Absolutely continuous functions of one variable are admissible transformations for the change of variables in the Lebesgue integral. Recently J. Malý [5] introduced a class of $n$-absolutely continuous functions giving an $n$-dimensional analog of the notion of absolute continuity from this point of view.

Here we consider a more general definition of absolutely continuous functions suggested by L. Zajíček. Suppose that $\Omega \subset \mathbb{R}^{n}$ is an open set and $0<\lambda \leq 1$. We say that a function $f: \Omega \rightarrow \mathbb{R}^{m}$ is $n, \lambda$-absolutely continuous if for each $\varepsilon>0$ there is $\delta>0$ such that for each disjoint finite family $\left\{B\left(x_{i}, r_{i}\right)\right\}$ of balls in $\Omega$ we have

$$
\sum_{i} \mathcal{L}_{n}\left(B\left(x_{i}, r_{i}\right)\right)<\delta \Rightarrow \sum_{i}\left(\operatorname{osc}_{B\left(x_{i}, \lambda r_{i}\right)} f\right)^{n}<\varepsilon .
$$

Absolute continuity from [5] coincides with $n$, 1-absolute continuity. In Section 3 we show that $n, \lambda$-absolute continuity implies continuity, weak differentiability with gradient in $L^{n}$, differentiability almost everywhere and the formula of change of variables. We also prove that the $n, \lambda_{1}$-absolutely continuous functions coincide with the $n, \lambda_{2}$-absolutely continuous functions for $0<\lambda_{1}<\lambda_{2}<1$.

In Section 4 we recall the result of M. Csörnyei from [1]. She proved that 2,1-absolutely continuous functions with respect to balls are not the same

2000 Mathematics Subject Classification: Primary 26B30; Secondary 26B10.

The research is supported by the grant GAČR 201/00/0767 and MSM 113200007. 
as 2,1-absolutely continuous functions with respect to cubes. We prove that $n, \lambda$-absolute continuity is the same for balls and cubes for $0<\lambda<1$.

Section 5 concerns a condition equivalent to $n, \lambda$-absolute continuity. We call it the Rado-Reichelderfer condition $\left(R R_{\lambda}\right)$.

In Section 6 we give an example of a function which is not $n, 1$-absolutely continuous but which is $n, \lambda$-absolutely continuous for any $0<\lambda<1$.

It is easy to see that $n, 1$-absolute continuity implies $n, \lambda$-absolute continuity for $0<\lambda<1$. Therefore all the subspaces of $n, 1$-absolutely continuous functions are also subspaces of $n, \lambda$-absolutely continuous functions. It was shown in [5] that $W^{1, n}$ mappings with finite dilatation and continuous $W^{1, n}$ pseudomonotone mappings are $n, 1$-absolutely continuous. Another interesting result concerning embedding into absolutely continuous functions can be found in J. Kauhanen, P. Koskela and J. Malý [4]. It is shown there that $\nabla f \in L^{n, 1}$ guarantees that $f$ has an $n$, 1-absolutely continuous representative. The symbol $L^{n, 1}$ denotes the Lorentz space.

2. Preliminaries. We denote by $\mathcal{L}_{n}$ the $n$-dimensional Lebesgue measure and by $\mathcal{H}^{n}$ the $n$-dimensional Hausdorff measure. Recall that the construction of the $n$-dimensional Hausdorff measure employs a scale of outer measures usually denoted by $\mathcal{H}_{\varepsilon}^{n}$.

We use the letter $\lambda$ for a real number $0<\lambda \leq 1$. Throughout the paper we consider an open set $\Omega \subset \mathbb{R}^{n}, n>1$.

We denote by $B(x, r)$ the $n$-dimensional open ball with center $x$ and diameter $r$ and by $\overline{B(x, r)}$ the corresponding closed ball. We write $Q(x, r)$ for the open cube with sides parallel to coordinate axes and with center $x$ and side length $2 r$. Throughout the paper we reserve the letter $B$ for balls and $Q$ for cubes. Given $B=B(x, r)$ we write $\lambda B=B(x, \lambda r)$.

The characteristic function of a set $A \subset \mathbb{R}^{n}$ is denoted by $\chi_{A}$. We use the notation $\left[x_{1}, x_{2}\right]$ for a point $x \in \mathbb{R}^{2}$.

We write $\operatorname{osc}_{A} f$ for the oscillation of $f: \Omega \rightarrow \mathbb{R}^{m}$ over the set $A \subset \Omega$, which is the diameter of $f(A)$.

Let $C_{\mathrm{c}}(\Omega)$ be the set of all continuous functions $f: \Omega \rightarrow \mathbb{R}$ with compact support. We denote by $\|\ldots\|$ the supremum norm on this space.

Let $f: \Omega \rightarrow \mathbb{R}^{m}$ be a mapping. We denote by $f^{\prime}(x)$ the Jacobi matrix of all partial derivatives of $f$ at $x$. We use the notation $\nabla f$ for the weak (distributional) derivative. If $m \geq n$, we denote by $J_{f}(x)$ the $\left(\begin{array}{l}m \\ n\end{array}\right)$-dimensional vector of all $n \times n$ minors of the Jacobi matrix $f^{\prime}(x)$.

We use the convention that $C$ denotes a generic positive constant which may change from expression to expression.

Now for the convenience of the reader we state the covering theorem we will use. 
TheOREm 2.1 (Vitali). Let $\mathcal{B}$ be an arbitrary family of balls in $\mathbb{R}^{n}$ with

$$
\sup \{\operatorname{diam} B: B \in \mathcal{B}\}<\infty .
$$

Then there exists a disjoint (at most countable) subsystem $\left\{B\left(x_{i}, r_{i}\right)\right\} \subset \mathcal{B}$ such that

$$
\bigcup_{B \in \mathcal{B}} B \subset \bigcup B\left(x_{i}, 5 r_{i}\right)
$$

3. Properties of $n, \lambda$-absolutely continuous functions. Given a function $f: \Omega \rightarrow \mathbb{R}^{m}$ and a measurable set $A \subset \Omega$, we define the $n, \lambda$ variation of $f$ on $A$ by

$$
\begin{aligned}
& V_{\lambda}^{n}(f, A)=\sup \left\{\sum_{i} \operatorname{osc}_{B\left(x_{i}, \lambda r_{i}\right)}^{n} f:\right. \\
& \left.\qquad\left\{B\left(x_{i}, r_{i}\right)\right\} \text { is a disjoint family of balls in } A\right\} .
\end{aligned}
$$

We denote by $B V_{\lambda}^{n}(\Omega)$ the class of all functions $f$ such that $V_{\lambda}^{n}(f, \Omega)<\infty$. We define $A C_{\lambda}^{n}(\Omega)$ as the family of all $n, \lambda$-absolutely continuous functions in $B V_{\lambda}^{n}(\Omega)$.

REMARK 3.1. It is easy to see that for $n=1$ and $0<\lambda \leq 1$ a function is $n, \lambda$-absolutely continuous if and only if it is absolutely continuous in the classical sense.

The following theorem states that in fact there are only two classes of $n, \lambda$-absolutely continuous functions for $n \geq 2$, namely those corresponding to the cases $\lambda=1$ and $0<\lambda<1$. Clearly $A C_{1}^{n} \subset A C_{\lambda}^{n}$ and in Section 6 we prove that $A C_{1}^{n} \neq A C_{\lambda}^{n}$ for $0<\lambda<1$ and $n \geq 2$.

Theorem 3.2. Let $0<\lambda_{1}<\lambda_{2}<1$ and $f: \Omega \rightarrow \mathbb{R}^{m}$. Then

(i) $f$ is $n, \lambda_{1}$-absolutely continuous if and only if $f$ is $n, \lambda_{2}$-absolutely continuous,

(ii) $B V_{\lambda_{1}}^{n}(\Omega)=B V_{\lambda_{2}}^{n}(\Omega)$,

(iii) $A C_{\lambda_{1}}^{n}(\Omega)=A C_{\lambda_{2}}^{n}(\Omega)$.

Proof. (i) It is clear that $n, \lambda_{2}$-absolute continuity implies $n, \lambda_{1}$-absolute continuity. Now, suppose that $f$ is $n, \lambda_{1}$-absolutely continuous. Fix $d \in \mathbb{N}$ such that $d>\lambda_{2} /\left(\left(1-\lambda_{2}\right) \lambda_{1}\right)$. Choose $\varepsilon>0$ and find $\delta>0$ such that for each disjoint finite family $\left\{B\left(\widetilde{x}_{i}, \widetilde{r}_{i}\right)\right\}$ of balls in $\Omega$ we have

$$
\sum_{i} \mathcal{L}_{n}\left(B\left(\widetilde{x}_{i}, \widetilde{r}_{i}\right)\right)<\delta \Rightarrow \sum_{i} \operatorname{osc}_{B\left(\widetilde{x}_{i}, \lambda_{1} \tilde{r}_{i}\right)}^{n} f<\frac{\varepsilon}{(d+1)^{n}} .
$$

Let $\left\{B\left(x_{i}, r_{i}\right)\right\}$ be a disjoint finite family of balls in $\Omega$ such that

$$
\sum_{i} \mathcal{L}_{n}\left(B\left(x_{i}, r_{i}\right)\right)<\delta
$$


There exist $a_{i}, b_{i} \in \overline{B\left(x_{i}, \lambda_{2} r_{i}\right)}$ such that $\operatorname{osc}_{B\left(x_{i}, \lambda_{2} r_{i}\right)} f=\left|f\left(a_{i}\right)-f\left(b_{i}\right)\right|$. Clearly

$$
\operatorname{osc}_{B\left(x_{i}, \lambda_{2} r_{i}\right)} f=\left|f\left(a_{i}\right)-f\left(b_{i}\right)\right| \leq \sum_{j=0}^{d} \operatorname{osc}_{B\left(b_{i}+(j / d)\left(a_{i}-b_{i}\right), \lambda_{2} r_{i} / d\right)} f .
$$

Hence there exists $j_{i} \in\{0, \ldots, d\}$ such that for

$$
B\left(\widetilde{x}_{i}, \widetilde{r}_{i}\right)=B\left(b_{i}+\frac{j_{i}}{d}\left(a_{i}-b_{i}\right), \frac{1}{\lambda_{1}} \cdot \frac{\lambda_{2} r_{i}}{d}\right)
$$

we have

$$
\operatorname{Osc}_{B\left(x_{i}, \lambda_{2} r_{i}\right)} f \leq(d+1) \operatorname{osc}_{B\left(\tilde{x}_{i}, \lambda_{1} \widetilde{r}_{i}\right)} f .
$$

Since $d>\lambda_{2} /\left(\left(1-\lambda_{2}\right) \lambda_{1}\right)$ we obtain $B\left(\widetilde{x}_{i}, \widetilde{r}_{i}\right) \subset B\left(x_{i}, r_{i}\right)$ and hence the balls $B\left(\widetilde{x}_{i}, \widetilde{r}_{i}\right)$ are pairwise disjoint. Clearly

$$
\sum_{i} \mathcal{L}_{n}\left(B\left(\widetilde{x}_{i}, \widetilde{r}_{i}\right)\right) \leq \sum_{i} \mathcal{L}_{n}\left(B\left(x_{i}, r_{i}\right)\right)<\delta .
$$

Thus (3.2) and (3.1) give

$$
\sum_{i} \operatorname{osc}_{B\left(x_{i}, \lambda_{2} r_{i}\right)}^{n} f \leq(d+1)^{n} \sum_{i} \operatorname{osc}_{B\left(\widetilde{x}_{i}, \lambda_{1} \tilde{r}_{i}\right)}^{n} f<(d+1)^{n} \frac{\varepsilon}{(d+1)^{n}}=\varepsilon .
$$

(ii) Clearly $V_{\lambda_{1}}^{n}(f, \Omega) \leq V_{\lambda_{2}}^{n}(f, \Omega)$. Let $\left\{B\left(x_{i}, r_{i}\right)\right\}$ be a disjoint family of balls in $\Omega$. Analogously to (i) we can find $B\left(\widetilde{x}_{i}, \widetilde{r}_{i}\right) \subset B\left(x_{i}, r_{i}\right)$ such that

$$
\sum_{i} \operatorname{osc}_{B\left(x_{i}, \lambda_{2} r_{i}\right)}^{n} f \leq(d+1)^{n} \sum_{i} \operatorname{osc}_{B\left(\widetilde{x}_{i}, \lambda_{1} \widetilde{r}_{i}\right)}^{n} f .
$$

It follows that $V_{\lambda_{2}}^{n}(f, \Omega) \leq(d+1)^{n} V_{\lambda_{1}}^{n}(f, \Omega)$.

(iii) This is a consequence of (i) and (ii).

Theorems 3.3-3.5 summarize nice properties of $n, \lambda$-absolutely continuous functions. These theorems were proved for $\lambda=1$ in [5].

The proofs of Theorems 3.3 and 3.4 are analogous to those in [5] and so we omit them.

Theorem 3.3. If $n \geq 2$, then $B V_{\lambda, \text { loc }}^{n}(\Omega) \subset W_{\text {loc }}^{1, n}(\Omega)$.

TheOREM 3.4. Let $f \in B V_{\lambda}^{n}(\Omega)$. Then $f$ is differentiable almost everywhere and $f^{\prime} \in L^{n}\left(\Omega, \mathbb{R}^{n}\right)$.

THEOREM 3.5. Let $f$ be an $n, \lambda$-absolutely continuous function on $\Omega$ with values in $\mathbb{R}^{m}, m \geq n$. Let $E$ be a measurable subset of $\Omega$ and $u$ be a measurable function on $E$ such that $u\left|J_{f}\right| \in L^{1}(E)$. Then

$$
\int_{E} u(x)\left|J_{f}(x)\right| d x=\int_{f(E)} \sum_{\{x \in E: f(x)=y\}} u(x) d \mathcal{H}^{n}(y) .
$$

Proof. The proof of this theorem in the case $\lambda=1$ is in [5]. Now, let $0<\lambda<1$. By Theorem 3.2 we can assume that $\lambda=1 / 2$. By Theorem 3.4 
each $n, 1 / 2$-absolutely continuous function is a.e. differentiable. Hence $[2$, Th. 3.1.8] shows that there is a sequence $\left\{f_{j}\right\}$ of Lipschitz functions from $\mathbb{R}^{n}$ to $\mathbb{R}^{m}$ such that

$$
\mathcal{L}_{n}\left(\Omega \backslash \bigcup_{j}\left\{x \in \Omega: f_{j}(x)=f(x) \text { and } f_{j}^{\prime}(x)=f^{\prime}(x)\right\}\right)=0 .
$$

Since (3.3) holds for Lipschitz mappings ([2, Th. 3.2.3]), we can assume that $\mathcal{L}_{n}(E)=0$.

Set

$$
\begin{aligned}
& E_{1}=\left\{x \in E: \liminf _{r \rightarrow 0} \frac{\operatorname{osc}_{B(x, r)} f}{\operatorname{osc}_{B(x, r / 2)} f} \leq 4\right\}, \\
& E_{2}=\left\{x \in E: \liminf _{r \rightarrow 0} \frac{\operatorname{osc}_{B(x, r)} f}{\operatorname{osc}_{B(x, r / 2)} f}>4\right\} .
\end{aligned}
$$

Choose $\varepsilon>0$ and take $\delta$ with $0<\delta<\varepsilon$ from the definition of $n, \lambda$-absolute continuity of $f$. Let $G \subset \Omega$ be an open set containing $E$ with $\mathcal{L}_{n}(G)<\delta$. For each $x \in E_{1}$ there is $r(x)>0$ such that

$$
\begin{aligned}
& B(x, r(x)) \subset G, \quad r(x)<\varepsilon / 30, \\
& \operatorname{osc}_{B(x, r(x) / 2)} f<\varepsilon / 30, \quad \operatorname{osc}_{B(x, r(x))} f<5 \operatorname{osc}_{B(x, r(x) / 2)} f .
\end{aligned}
$$

For every $x \in E_{2}$ there is $\omega(x)$ such that for all $r \in(0, \omega(x))$ we have

$$
\frac{\operatorname{osc}_{B(x, r)} f}{r}>2 \frac{\operatorname{osc}_{B(x, r / 2)} f}{r / 2} \text {. }
$$

Thus

$$
\lim _{r \rightarrow 0} \frac{\operatorname{osc}_{B(x, r)} f}{r}=0 .
$$

Hence for each $x \in E_{2}$ we can choose $r(x)>0$ such that

$$
\begin{aligned}
& B(x, r(x)) \subset G, \\
& \operatorname{osc}_{B(x, r(x) / 2)} f<r(x)<\varepsilon / 30, \quad \operatorname{osc}_{B(x, r(x))} f<r(x) .
\end{aligned}
$$

Put

$$
\varrho(x)=5 \operatorname{osc}_{B(x, r(x) / 2)} f+r(x) .
$$

We use Theorem 2.1 in $\mathbb{R}^{m}$ to find a disjoint system

$$
\left\{B\left(f\left(x_{i}\right), \varrho\left(x_{i}\right)\right)\right\} \subset\{B(f(x), \varrho(x)): x \in E\}
$$

such that

$$
f(E) \subset \bigcup_{i} B\left(f\left(x_{i}\right), 5 \varrho\left(x_{i}\right)\right)
$$

Thanks to (3.4) and (3.5), $f\left(B\left(x_{i}, r\left(x_{i}\right)\right)\right) \subset B\left(f\left(x_{i}\right), \varrho\left(x_{i}\right)\right)$. It follows that 
$B\left(x_{i}, r\left(x_{i}\right)\right)$ are pairwise disjoint and using (3.4) and (3.5) we obtain

$$
\begin{aligned}
\mathcal{H}_{\varepsilon}^{n}(f(E)) & \leq C \sum_{i} \varrho\left(x_{i}\right)^{n} \\
& \leq C \sum_{i}\left(\operatorname{osc}_{B\left(x_{i}, r\left(x_{i}\right) / 2\right)}^{n} f+r\left(x_{i}\right)^{n}\right) \leq C \varepsilon+C \delta \leq C \varepsilon .
\end{aligned}
$$

Letting $\varepsilon \rightarrow 0$ we obtain $\mathcal{H}^{n}(f(E))=0$.

4. Different properties of $A C_{1}^{n}$ and $A C_{\lambda}^{n}, 0<\lambda<1$. We denote by $\mathcal{Q}$ the set of all cubes in $\mathbb{R}^{n}$ with sides parallel to coordinate axes. We say that a function $f: \Omega \rightarrow \mathbb{R}^{m}$ is $\mathcal{Q}, n, \lambda$-absolutely continuous if for each $\varepsilon>0$ there is $\delta>0$ such that for each disjoint finite family $\left\{Q\left(x_{i}, r_{i}\right)\right\}$ of cubes in $\Omega$ we have

$$
\sum_{i} \mathcal{L}_{n}\left(Q\left(x_{i}, r_{i}\right)\right)<\delta \Rightarrow \sum_{i} \operatorname{osc}_{Q\left(x_{i}, \lambda r_{i}\right)}^{n} f<\varepsilon .
$$

Analogously to Section 2 we define $\mathcal{Q}-B V_{\lambda}^{n}(\Omega)$ and $\mathcal{Q}-A C_{\lambda}^{n}(\Omega)$.

The next theorem from [1] states that 2,1-absolutely continuous functions are not the same as $\mathcal{Q}, 2,1$-absolutely continuous functions.

Theorem 4.1. There exists a function $f: \mathbb{R}^{2} \rightarrow \mathbb{R}$ such that $f \in$ $A C_{1}^{2}\left(\mathbb{R}^{2}\right)$ but $f \notin \mathcal{Q}-A C_{1}^{2}\left(\mathbb{R}^{2}\right)$.

In contrast to Theorem 4.1 there is no difference between $A C_{\lambda}^{n}$ and $\mathcal{Q}-A C_{\lambda}^{n}$ for $0<\lambda<1$.

Theorem 4.2. Let $0<\lambda<1$. Then $A C_{\lambda}^{n}(\Omega)=\mathcal{Q}-A C_{\lambda}^{n}(\Omega)$.

Proof. Let $\left\{B\left(x_{i}, r_{i}\right)\right\}$ be a disjoint family of balls in $\Omega$. Put $\widetilde{r}_{i}=r_{i} / n$. Clearly

$$
B\left(x_{i}, \lambda r_{i} / n\right) \subset Q\left(x_{i}, \lambda \widetilde{r}_{i}\right) \text { and } Q\left(x_{i}, \widetilde{r}_{i}\right) \subset B\left(x_{i}, r_{i}\right) .
$$

Hence $Q\left(x_{i}, \widetilde{r}_{i}\right)$ are pairwise disjoint and $\operatorname{osc}_{B\left(x_{i}, \lambda r_{i} / n\right)} f \leq \operatorname{osc}_{Q\left(x_{i}, \lambda \widetilde{r}_{i}\right)} f$. Thus $\mathcal{Q}-A C_{\lambda}^{n} \subset A C_{\lambda / n}^{n}(\Omega)$. Therefore Theorem 3.2 shows that

$$
\mathcal{Q}-A C_{\lambda}^{n} \subset A C_{\lambda}^{n}(\Omega) \text {. }
$$

It is easy to see that an analog of Theorem 3.2 holds also for $\mathcal{Q}-A C_{\lambda}^{n}$. That is, $\mathcal{Q}-A C_{\lambda_{1}}^{n}=\mathcal{Q}-A C_{\lambda_{2}}^{n}$ for $0<\lambda_{1}<\lambda_{2}<1$. Together with

$$
Q(x, \lambda r / n) \subset B(x, \lambda r) \subset B(x, r) \subset Q(x, r),
$$

this implies that $A C_{\lambda}^{n}(\Omega) \subset \mathcal{Q}-A C_{\lambda}^{n}(\Omega)$.

REMARK 4.3. An analogous proof works not only for cubes but also for the family $\mathcal{K}=\left\{a+b K_{0}: a \in \mathbb{R}^{n}, b \in \mathbb{R}^{+}\right\}$where $K_{0}$ is a fixed bounded open convex set of non-empty interior. Hence the definition of $A C_{\lambda}^{n}$ does not depend on the shape of the "balls" for $0<\lambda<1$. 
The next theorem shows that $A C_{\lambda}^{n}$ functions are stable under bilipschitz mappings for $0<\lambda<1$. This is also not true for $n$, 1 -absolutely continuous functions (see [3] for details).

Theorem 4.4. Let $f: \Omega \rightarrow \mathbb{R}^{m}$ be a function and let $0<\lambda<1$. Let $F: \Omega \rightarrow \mathbb{R}^{n}$ be a bilipschitz mapping. Then

$$
f \in A C_{\lambda}^{n}(\Omega) \Leftrightarrow f \circ F^{-1} \in A C_{\lambda}^{n}(F(\Omega)) .
$$

Proof. It is enough to prove one implication. Let $f \in A C_{\lambda}^{n}(\Omega)$. Let $L$ be the bilipschitz constant for $F$, i.e.

$$
|x-y| / L \leq|F(x)-F(y)| \leq L|x-y| .
$$

Choose $\varepsilon$ and take $\delta_{f}$ as in the definition of absolute continuity of $f$. Put $\delta=\delta_{f} / L^{n}$. Let $\left\{B\left(x_{i}, r_{i}\right)\right\}$ be a disjoint family of balls in $F(\Omega)$ such that $\sum_{i} \mathcal{L}_{n}\left(B\left(x_{i}, r_{i}\right)\right)<\delta$. Set $\widetilde{r}_{i}=r_{i} / L$. Clearly

$$
\begin{aligned}
F^{-1}\left(B\left(x_{i}, \lambda r_{i} / L^{2}\right)\right) & \subset B\left(F^{-1}\left(x_{i}\right), \lambda \widetilde{r}_{i}\right), \\
B\left(F^{-1}\left(x_{i}\right), \widetilde{r}_{i}\right) & \subset F^{-1}\left(B\left(x_{i}, r_{i}\right)\right) .
\end{aligned}
$$

It follows that the $B\left(F^{-1}\left(x_{i}\right), \widetilde{r}_{i}\right)$ are pairwise disjoint and

$$
\sum_{i} \mathcal{L}_{n}\left(B\left(F^{-1}\left(x_{i}\right), \widetilde{r}_{i}\right)\right)=L^{n} \sum_{i} \mathcal{L}_{n}\left(B\left(x_{i}, r_{i}\right)\right)<L^{n} \delta=\delta_{f}
$$

Hence

$$
\begin{aligned}
\sum_{i} \operatorname{osc}_{B\left(x_{i}, \lambda r_{i} / L^{2}\right)}^{n} f \circ F^{-1} & =\sum_{i} \operatorname{osc}_{F}^{n}\left(B\left(x_{i}, \lambda r_{i} / L^{2}\right)\right) \\
& \leq \sum_{i} \operatorname{osc}_{B\left(F^{-1}\left(x_{i}\right), \lambda \tilde{r}_{i}\right)}^{n} f \leq \varepsilon .
\end{aligned}
$$

This proves $f \circ F^{-1} \in A C_{\lambda /\left(2 L^{2}\right)}^{n}(F(\Omega))$. By Theorem 3.2, $f \circ F^{-1} \in$ $A C_{\lambda}^{n}(F(\Omega))$ as well.

5. Relation between $A C_{\lambda}^{n}$ and $R R_{\lambda}$-conditions. We say that a func-

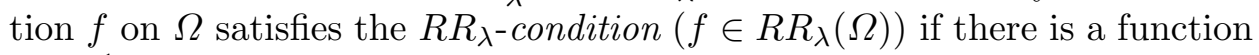
$g \in L^{1}(\Omega)$, called a weight, such that

$$
\operatorname{osc}_{B(x, \lambda r)}^{n} f \leq \int_{B(x, r)} g(y) d y \quad \text { for every ball } B(x, r) \subset \Omega .
$$

We say that a function $f$ on $\Omega$ satisfies the $R R_{\lambda}^{\star}$-condition $\left(f \in R R_{\lambda}^{\star}(\Omega)\right)$ if there is a finite (not necessarily absolutely continuous) Borel measure $\mu$ such that

$$
\operatorname{osc}_{B(x, \lambda r)}^{n} f \leq \mu(B(x, r)) \quad \text { for every ball } B(x, r) \subset \Omega .
$$

A condition similar to $R R_{1}$ was used by Rado and Reichelderfer [7] as a sufficient condition for the area formula and for differentiability a.e. 
Theorems 5.1 and 5.2 show the connection between the $R R$-conditions and absolutely continuous functions of several variables.

TheOREm 5.1. Let $\Omega \subset \mathbb{R}^{n}$ be an open set and $0<\lambda \leq 1$. Then $R R_{\lambda}(\Omega)=A C_{\lambda}^{n}(\Omega)$.

TheOREm 5.2. Let $\Omega \subset \mathbb{R}^{n}$ be an open set and $0<\lambda \leq 1$. Then $R R_{\lambda}^{\star}(\Omega)=B V_{\lambda}^{n}(\Omega)$.

Given $f_{0} \in B V_{1 / 2}^{n}(\Omega)$ we define

$$
\begin{aligned}
& V^{\star}(f)=\sup \left\{\sum_{i=1}^{k} \alpha_{i} \operatorname{osc}_{\frac{1}{2} B_{i}}^{n} f_{0}: k \in \mathbb{N}, \sum_{i=1}^{k} \alpha_{i} \chi_{B_{i}} \leq f,\right. \\
& \left.\alpha_{i} \in \mathbb{Q}, \alpha_{i} \geq 0\right\}
\end{aligned}
$$

for every non-negative function $f \in C_{\mathrm{c}}(\Omega)$. It is easy to see that for every $\lambda>0$ and non-negative $f, f_{1}, f_{2} \in C_{\mathrm{c}}(\Omega)$ we have

$$
V^{\star}(\lambda f)=\lambda V^{\star}(f) \text { and } V^{\star}\left(f_{1}+f_{2}\right) \geq V^{\star}\left(f_{1}\right)+V^{\star}\left(f_{2}\right) .
$$

For every measurable set $A \subset \Omega$ we have $f \geq \chi_{A} \Rightarrow V^{\star}(f) \geq V_{1 / 2}^{n}\left(f_{0}, A\right)$.

We will need the following consequence of the Besicovitch Covering Theorem from [1].

Lemma 5.3. There exists a constant $c$ such that for every $r \in \mathbb{N}$, each family $\mathcal{B}_{0}$ of balls satisfying $\sum_{B \in \mathcal{B}_{0}} \chi_{B} \leq r$ (i.e. each point of $\mathbb{R}^{n}$ is covered by at most $r$ elements of $\mathcal{B}_{0}$ ) can be partitioned into cr classes of pairwise disjoint elements of $\mathcal{B}_{0}$.

Lemma 5.4. Let $f_{0} \in B V_{1 / 2}^{n}(\Omega)$. Define $V^{\star}$ by (5.1). Then there exists a constant $c>0$ such that for every non-negative $f \in C_{\mathrm{c}}(\Omega)$ we have

$$
V^{\star}(f) \leq c\|f\| V_{1 / 2}^{n}\left(f_{0}, \Omega\right) .
$$

Proof. It is enough to prove that

$$
\sum_{i=1}^{k} \alpha_{i} \chi_{B\left(x_{i}, r_{i}\right)} \leq\|f\| \cdot \chi_{\Omega} \Rightarrow \sum_{i=1}^{k} \alpha_{i} \operatorname{osc}_{B\left(x_{i}, r_{i} / 2\right)}^{n} f_{0}<c\|f\| V_{1 / 2}^{n}\left(f_{0}, \Omega\right)
$$

for every $k \in \mathbb{N}$ and for every sequence of non-negative rational numbers $\alpha_{i}$. We can replace $\alpha_{i}$ by $N \alpha_{i}$ and $f$ by $N f$ for every $N \in \mathbb{N}$. Hence we can assume that all the numbers $\alpha_{i}$ are integers. By replacing $k$ by $\widetilde{k}=\sum_{i=1}^{k} \alpha_{i}$ we can suppose that $\alpha_{i}=1$ for every $i$. Thus it is enough to prove that

$$
\sum_{i=1}^{k} \chi_{B\left(x_{i}, r_{i}\right)} \leq\|f\| \cdot \chi_{\Omega} \Rightarrow \sum_{i=1}^{k} \operatorname{osc}_{B\left(x_{i}, r_{i} / 2\right)}^{n} f_{0}<c\|f\| V_{1 / 2}^{n}\left(f_{0}, \Omega\right) .
$$

Applying Lemma 5.3 we can divide $\left\{B\left(x_{i}, r_{i}\right)\right\}_{i=1}^{k}$ into $c\|f\|$ classes of pairwise disjoint elements and for pairwise disjoint balls the sum of the $n$th powers of the oscillations over their $\frac{1}{2}$ multiples is at most $V_{1 / 2}^{n}\left(f_{0}, \Omega\right)$. 
Proof of Theorem 5.2. The proof of $R R_{\lambda}^{\star}(\Omega) \subset B V_{\lambda}^{n}(\Omega)$ is not difficult and analogous to the proof of $R R_{1}(\Omega) \subset A C_{1}^{n}(\Omega)$ from [5]. Thus we omit it.

The proof of

$$
B V_{\lambda}^{n}(\Omega) \subset R R_{\lambda}^{\star}(\Omega)
$$

was given in [1] in the case $\lambda=1$ and $\Omega=\mathbb{R}^{n}$. Our proof is analogous to [1] so we skip some details. We give the proof of (5.2) only in the case $0<\lambda<1$. The case $\lambda=1$ is analogous so we omit it. By Theorem 3.2 we can suppose that $\lambda=1 / 2$.

Fix $f_{0} \in B V_{1 / 2}^{n}(\Omega)$ and define $V^{\star}$ by (5.1). Thanks to Lemma 5.4 the sets

$$
\begin{aligned}
& F_{1}=\left\{f \in C_{\mathrm{c}}(\Omega): f \geq 0, V^{\star}(f)>1\right\}, \\
& F_{2}=\left\{f \in C_{\mathrm{c}}(\Omega): c\left\|f^{+}\right\| V_{1 / 2}^{n}\left(f_{0}, \Omega\right)<1\right\}
\end{aligned}
$$

are disjoint convex subsets of $C_{\mathrm{c}}(\Omega)$ and $F_{2}$ has non-empty interior. By the Hahn-Banach theorem there is a bounded linear functional $\mathcal{F}$ such that $\mathcal{F}(f) \geq 1$ for every $f \in F_{1}$ and $\mathcal{F}(f) \leq 1$ for every $f \in F_{2}$. There is a Radon measure $\mu$ such that $\mathcal{F}(f)=\int_{\Omega} f d \mu$ by the Riesz representation theorem. This measure is finite since $\mathcal{F}(f) \leq 1$ for every $f \in F_{2}$. Hence

$$
V^{\star}(f) \geq 1 \Rightarrow \int_{\Omega} f d \mu \geq 1
$$

for every non-negative $f \in C_{\mathrm{c}}(\Omega)$. Due to linearity of $\mathcal{F}$, for every nonnegative $f \in C_{\mathrm{c}}(\Omega)$ with $V^{\star}(f)>0$ we have $\int_{\Omega} f d \mu \geq V^{\star}(f)$.

Suppose that $V^{\star}(f)=0$ for a non-negative $f \in C_{\mathrm{c}}(\Omega)$. If $f_{0}$ is locally constant then the statement of Theorem 5.2 is obvious. Otherwise there exists $f_{1}$ such that $V^{\star}\left(f_{1}\right)>0$. Hence $V^{\star}\left(f+\varepsilon f_{1}\right)>0$ and $\int_{\Omega}\left(f+\varepsilon f_{1}\right) d \mu>0$ for all $\varepsilon>0$. This gives $\int_{\Omega} f d \mu \geq 0$. So $\mu$ is a non-negative measure and

$$
\int_{\Omega} f d \mu \geq V^{\star}(f)
$$

for every non-negative $f \in C_{\mathrm{c}}(\Omega)$.

Therefore $f \geq \chi_{B} \Rightarrow V^{\star}(f) \geq V_{1 / 2}^{n}\left(f_{0}, B\right)$ shows that $\mu(B) \geq V_{1 / 2}^{n}\left(f_{0}, B\right)$ for every ball $B \subset \Omega$. Hence

$$
\mu(B) \geq V_{1 / 2}^{n}\left(f_{0}, B\right) \geq \operatorname{osc}_{\frac{1}{2} B}^{n} f_{0}
$$

and therefore $f_{0} \in R R_{1 / 2}^{\star}(\Omega)$.

We will need the following consequence of the Besicovitch Covering Theorem from [1].

Lemma 5.5. Let $\mathcal{B}_{0}$ be a family of balls in $\mathbb{R}^{n}$. Assume that for every $B(x, r) \in \mathcal{B}_{0}$ there exists a point $O_{B} \in B(x, r / 2)$ which is covered by at 
most $r$ elements of $\mathcal{B}_{0}$. Then there exists a constant $c$ such that $\mathcal{B}_{0}$ can be partitioned into $\mathrm{cr}$ classes of pairwise disjoint elements of $\mathcal{B}_{0}$.

Proof of Theorem 5.1. The proof of $R R_{\lambda}(\Omega) \subset A C_{\lambda}^{n}(\Omega)$ was given in the case $\lambda=1$ in [5]. The general case is analogous and not difficult and therefore we omit it.

The proof of

$$
A C_{\lambda}^{n}(\Omega) \subset R R_{\lambda}(\Omega)
$$

was given in [1] in the case $\lambda=1$ and $\Omega=\mathbb{R}^{n}$. Our proof is analogous so we skip some details. We give the proof of (5.3) only in the case $0<\lambda<1$. The case $\lambda=1$ is analogous. By Theorem 3.2 we can suppose that $\lambda=1 / 2$.

Fix $f_{0} \in A C_{1 / 2}^{n}(\Omega)$ and define $V^{\star}$ by (5.1).

For every $\varepsilon>0$ there exists $\delta>0$ such that for every measurable set $A \subset \Omega$ we have $\mathcal{L}_{n}(A)<\delta \Rightarrow V_{1 / 2}^{n}\left(f_{0}, A\right)<\varepsilon$. That is, for every $\delta \in[0, \infty]$ there is $\varepsilon(\delta)$ such that $\mathcal{L}_{n}(A)<\delta \Rightarrow V_{1 / 2}^{n}\left(f_{0}, A\right)<\varepsilon(\delta)$ and $\lim _{\delta \rightarrow 0} \varepsilon(\delta)=$ $\varepsilon(0)=0$. We can also assume that the function $\varepsilon(\delta)$ is increasing, concave and bounded by $2 V_{1 / 2}^{n}\left(f_{0}, \Omega\right)$.

For every $f \in C_{\mathrm{c}}(\Omega)$ we define

$$
\begin{aligned}
& V^{\star \star}(f)=\inf \left\{\sum_{i=1}^{k} \alpha_{i} \varepsilon\left(\mathcal{L}_{n}\left(A_{i}\right)\right): \sum_{i=1}^{k} \alpha_{i} \chi_{A_{i}} \geq f^{+},\right. \\
& \left.k \in \mathbb{N}, \alpha_{i} \in \mathbb{Q}, \alpha_{i} \geq 0\right\} .
\end{aligned}
$$

It is clear that for all $\lambda>0$ and $f, f_{1}, f_{2} \in C_{\mathrm{c}}(\Omega)$ we have

$$
V^{\star \star}(\lambda f)=\lambda V^{\star \star}(f) \text { and } V^{\star \star}\left(f_{1}+f_{2}\right) \leq V^{\star \star}\left(f_{1}\right)+V^{\star \star}\left(f_{2}\right) .
$$

Clearly for every measurable set $A \subset \Omega$ we obtain $0 \leq f \leq \chi_{A} \Rightarrow V^{\star \star}(f) \leq$ $\varepsilon\left(\mathcal{L}_{n}(A)\right)$. By Lemma 5.6 below, there exists a constant $c^{\prime}>0$ such that for every non-negative $f \in C_{\mathrm{c}}(\Omega)$ we have

$$
V^{\star}(f) \leq c^{\prime} V^{\star \star}(f) .
$$

Hence the sets

$$
F_{1}=\left\{f \in C_{\mathrm{c}}(\Omega): f \geq 0, V^{\star}(f)>1\right\}, \quad F_{2}=\left\{f \in C_{\mathrm{c}}(\Omega): c^{\prime} V^{\star \star}(f)<1\right\}
$$

are disjoint convex subsets of $C_{\mathrm{c}}(\Omega)$. In addition, $F_{2}$ has non-empty interior, because $V^{\star \star}(f) \leq 2 V_{1 / 2}^{n}\left(f_{0}, \Omega\right)\|f\|$. Therefore there exists a bounded linear functional $\mathcal{F}$ such that $\mathcal{F}(f) \geq 1$ for every $f \in F_{1}$ and $\mathcal{F}(f) \leq 1$ for every $f \in F_{2}$. Hence there exists a non-negative Radon measure $\mu$ such that for every non-negative $f \in C_{\mathrm{c}}(\Omega)$ we obtain

$$
V^{\star}(f) \leq \int_{\Omega} f d \mu \leq c^{\prime} V^{\star \star}(f) .
$$


We know that $f \geq \chi_{A} \Rightarrow V^{\star}(f) \geq V_{1 / 2}^{n}\left(f_{0}, A\right)$ for every measurable set $A \subset \Omega$. Hence for every ball $B \subset \Omega$,

$$
\operatorname{osc}_{\frac{1}{2} B}^{n} f_{0} \leq \mu(B) \text {. }
$$

For every measurable set $A \subset \Omega$ it is true that $0 \leq f \leq \chi_{A} \Rightarrow V^{\star \star}(f) \leq$ $\varepsilon\left(\mathcal{L}_{n}(A)\right)$. Hence $\int_{\Omega} f d \mu \leq c^{\prime} V^{\star \star}(f)$ gives $\mu(A) \leq c^{\prime} \varepsilon\left(\mathcal{L}_{n}(A)\right) \leq 2 c^{\prime} V_{1 / 2}^{n}\left(f_{0}, \Omega\right)$ for every measurable set $A \subset \Omega$. Thus $\mu$ is a finite absolutely continuous measure. Together with (5.5), this implies that $f_{0} \in R R_{1 / 2}(\Omega)$.

Lemma 5.6. Let $f_{0} \in A C_{\lambda}^{n}(\Omega)$. Define $V^{\star}$ and $V^{\star \star}$ by (5.1) and (5.4). Then there exists a constant $c^{\prime}>0$ such that for every non-negative $f \in$ $C_{\mathrm{c}}(\Omega)$ we have

$$
V^{\star}(f) \leq c^{\prime} V^{\star \star}(f) .
$$

Proof. It is enough to show that

$$
\sum_{i=1}^{k} \alpha_{i} \chi_{B_{i}} \leq \sum_{j=1}^{l} \beta_{j} \chi_{A_{j}} \Rightarrow \sum_{i=1}^{k} \alpha_{i} \operatorname{Osc}_{\frac{1}{2} B_{i}}^{n} f_{0} \leq c^{\prime} \sum_{j=1}^{l} \beta_{j} \varepsilon\left(\mathcal{L}_{n}\left(A_{j}\right)\right)
$$

for every measurable sets $A_{1}, \ldots, A_{l}$ and non-negative rational numbers $\alpha_{i}, \beta_{j}$. As before, we can assume that all the $\alpha_{i}, \beta_{j}$ are integers, and even that $\alpha_{i}=\beta_{j}=1$. Hence it is enough to prove that

$$
\sum_{i=1}^{k} \chi_{B_{i}} \leq \sum_{j=1}^{l} \chi_{A_{j}} \Rightarrow \sum_{i=1}^{k} \operatorname{osc}_{\frac{1}{2} B_{i}}^{n} f_{0} \leq c^{\prime} \sum_{j=1}^{l} \varepsilon\left(\mathcal{L}_{n}\left(A_{j}\right)\right) .
$$

For every $i<j$ we can replace $A_{i}, A_{j}$ by $\widetilde{A}_{i}=A_{i} \cup A_{j}$ and $\widetilde{A}_{j}=A_{i} \cap A_{j}$. This does not change $\sum_{j=1}^{l} \chi_{A_{j}}$ and decreases $\sum_{j=1}^{l} \varepsilon\left(\lambda\left(A_{j}\right)\right)$, thanks to concavity of $\varepsilon(\delta)$. After finitely many steps we obtain $A_{1} \supset \ldots \supset A_{l}$. Then clearly

$$
\sum_{i=1}^{k} \chi_{B_{i}} \leq \sum_{j=1}^{l} \chi_{A_{j}} \Rightarrow A_{j} \supset\left\{x: \sum_{i=1}^{k} \chi_{B_{i}} \geq j\right\} .
$$

So due to monotonicity of $\varepsilon(\delta)$ it is enough to prove that for every set $K_{0}=\left\{B_{1}, \ldots, B_{k}\right\}$ of balls and for

$$
\delta_{j}=\mathcal{L}_{n}\left(\left\{x \in \mathbb{R}^{n}: \sum_{B \in K_{0}} \chi_{B} \geq j\right\}\right)
$$

and $\varepsilon_{j}=\varepsilon\left(\delta_{j}\right)$ we have

$$
\sum_{B \in K_{0}} \operatorname{osc}_{\frac{1}{2} B}^{n} f_{0} \leq c^{\prime} \sum_{j \geq 1} \varepsilon_{j}
$$


Let $f$ denote $\sum_{B \in K_{0}} \chi_{B}$. For every $j \in \mathbb{N}$ we define

$$
K_{j}=\left\{B \in K_{0}: \min _{\frac{1}{2} B} f \in\left\langle 2^{j-1}, 2^{j}\right)\right\} .
$$

Then for every $B \in K_{j}$ there exists a point $O_{B} \in \frac{1}{2} B$ which is covered by at most $2^{j}-1$ balls from $\{B\}_{B \in K_{0}}$ and hence at most $2^{j}-1$ balls from $\{B\}_{B \in K_{j}}$. Hence Lemma 5.5 gives us a constant $c$ such that each $K_{j}$ can be divided into $\left(2^{j}-1\right) c$ classes $K_{j}^{1}, \ldots, K_{j}^{\left(2^{j}-1\right) c}$ such that

$$
B_{1}, B_{2} \in K_{j}^{i}, B_{1} \neq B_{2} \Rightarrow B_{1} \cap B_{2}=\emptyset .
$$

For every fixed $K_{j}^{i}$ we have

$$
\sum_{B \in K_{j}^{i}} \mathcal{L}_{n}(B)=2^{n} \sum_{B \in K_{j}^{i}} \mathcal{L}_{n}\left(\frac{1}{2} B\right)=2^{n} \mathcal{L}_{n}\left(\bigcup_{B \in K_{j}^{i}} \frac{1}{2} B\right)
$$

and by the definition of $K_{j}^{i}$ it is evident that

$$
\bigcup_{B \in K_{j}^{i}} \frac{1}{2} B \subset\left\{x: f(x) \geq 2^{j-1}\right\} .
$$

Therefore, since the balls in $K_{j}^{i}$ are pairwise disjoint,

$$
\sum_{B \in K_{j}^{i}} \operatorname{Osc}_{\frac{1}{2} B}^{n} f_{0} \leq \varepsilon\left(2^{n} \delta_{2^{j-1}}\right) \leq 2^{n} \varepsilon\left(\delta_{2^{j-1}}\right)=2^{n} \varepsilon_{2^{j-1}} .
$$

Together with $\varepsilon_{1} \geq \varepsilon_{2} \geq \ldots>0$ this implies that

$$
\begin{aligned}
\sum_{B \in K_{0}} \operatorname{osc}_{\frac{1}{2} B}^{n} f_{0} & \leq \sum_{j \geq 1} \sum_{i=1}^{\left(2^{j}-1\right) c} 2^{n} \varepsilon_{2^{j-1}}=2^{n} c \sum_{j \geq 1}\left(2^{j}-1\right) \varepsilon_{2^{j-1}} \\
& \leq 2^{n+1} c \sum_{j \geq 0} 2^{j} \varepsilon_{2^{j}} \leq 2^{n+2} c \sum_{j \geq 1} \varepsilon_{j} .
\end{aligned}
$$

6. Examples of $A C_{\lambda}^{n}$ and $B V_{\lambda}^{n}$ functions. The following theorem from [6] states that functions with bounded $n$, 1-variation have a continuous representative.

Theorem 6.1. Suppose that $n \geq 2$. Let $f \in B V_{1}^{n}(\Omega)$. Then $f$ has a continuous representative $\bar{f}$ and

$$
\lim _{y \rightarrow x} f(y)=\bar{f}(x) \quad \text { for each } x \in \Omega .
$$

TheOREM 6.2. Let $0<\lambda<1$ and $n=2$. Then there exists a function $f \in B V_{\lambda}^{2}(B(0,1 / 8))$ which does not have any continuous extension from $B(0,1 / 8) \backslash\{0\}$ to 0 . 
Proof. By Theorem 3.2 we can suppose that $\lambda=1 / 2$. Set

$$
g(t)=\frac{1}{t^{2} \log ^{2} t}, \quad t \in(0,1 / 8)
$$

We claim that the function

$$
f(x)= \begin{cases}\sin (\log (\log (1 /|x|))), & x \neq 0, x \in B(0,1 / 8), \\ 0, & x=0\end{cases}
$$

satisfies the $R R_{1 / 2}^{\star}$-condition with $\mu=2 \delta_{0}+C g(|x|) \mathcal{L}_{2}$, where $\delta_{0}$ denotes the Dirac measure at 0 . Clearly $\mu$ is a finite measure.

Let $B=B(c, r) \subset B(0,1 / 8)$. If $0 \in B$ then

$$
\operatorname{osc}_{\frac{1}{2} B}^{2} f \leq 2=2 \delta_{0}(B)<\mu(B) .
$$

Let $0 \notin B$. Then $r \leq|c|$. Clearly for all $x \in \frac{1}{2} B$ we have

$$
g(x) \geq C \frac{1}{|c|^{2} \log ^{2}|c|} .
$$

Hence

$$
\mu(B) \geq \int_{B} g(|x|) d x \geq C \int_{\frac{1}{2} B} \frac{1}{|c|^{2} \log ^{2}|c|} d x=C \frac{r^{2}}{|c|^{2} \log ^{2}|c|}
$$

On the other hand

$$
\begin{aligned}
\operatorname{Osc}_{\frac{1}{2} B} f(x) & \leq \operatorname{osc}_{\frac{1}{2} B} \log \left(\log \frac{1}{|x|}\right)=\operatorname{osc}_{t \in(c-r / 2, c+r / 2)} \log \left(\log \frac{1}{t}\right) \\
& \leq \int_{c-r / 2}^{c+r / 2} \frac{d t}{t \log \frac{1}{t}} \leq \frac{C r}{|c| \log \frac{1}{|c|}} .
\end{aligned}
$$

Hence (6.1) and (6.2) give us

$$
\operatorname{Osc}_{\frac{1}{2} B}^{2} f(x) \leq \mu(B) .
$$

Thus we have verified the $R R_{1 / 2}^{\star}$-condition for $f$ with $\mu$. Hence Theorem 5.2 shows that $f \in B V_{1 / 2}^{n}(B(0,1 / 8))$. Clearly there is no continuous extension of $f$ from $B(0,1 / 8) \backslash\{0\}$ to 0 .

Thanks to Theorems 3.2 and 6.2 it is easy to obtain the following corollary.

COROLlary 6.3. The function $f$ from Theorem 6.2 belongs to

$$
B V_{1 / 2}^{2}(B(0,1 / 8)) \backslash B V_{1}^{2}(B(0,1 / 8)) .
$$

THEOREM 6.4. Let $0<\lambda<1$ and $n=2$. There exists a function $f_{a} \in$ $A C_{\lambda}^{2}(B(0,1 / 8)) \backslash A C_{1}^{2}(B(0,1 / 8))$. 
Proof. By Theorem 3.2 we can suppose that $\lambda=1 / 2$. We use the functions $f$ and $g$ from the proof of Theorem 6.2. Set

$$
h(t)=\frac{1}{t}\left(\frac{1}{\log \left(\log \frac{1}{2 t}\right)}\right)^{\prime}=\frac{-1}{t^{2} \log (2 t) \log ^{2}\left(\log \frac{1}{2 t}\right)}, \quad t \in(0,1 / 8) .
$$

We claim that the function

$$
f_{a}(x)= \begin{cases}\frac{f(x)}{\sqrt{\log \left(\log \frac{1}{|x|}\right)}}=\frac{\sin \left(\log \left(\log \frac{1}{|x|}\right)\right)}{\sqrt{\log \left(\log \frac{1}{|x|}\right)},} & x \neq 0, x \in B(0,1 / 8), \\ 0, & x=0,\end{cases}
$$

satisfies the $R R_{1 / 2}$-condition with weight

$$
\theta=C h(|x|)+C g(|x|) .
$$

Clearly $\theta \in L^{1}(B(0,1 / 8))$.

Choose $B=B(c, r) \subset B(0,1 / 8) \subset \mathbb{R}^{2}$.

Let $0 \notin B$. The function $(\sin t) / \sqrt{t}$ is Lipschitz for $t \geq \log (\log 8)$. Hence (6.2) gives

$$
\begin{aligned}
\operatorname{osc}_{\frac{1}{2} B} f_{a} & \leq \operatorname{osc}\left\{\frac{\sin t}{\sqrt{t}}: \log \log \frac{1}{|c|+r / 2}<t<\log \log \frac{1}{|c|-r / 2}\right\} \\
& \leq C \operatorname{osc}_{t \in(c-r / 2, c+r / 2)} \log \left(\log \frac{1}{t}\right) \leq \frac{C r}{|c| \log \frac{1}{|c|}}
\end{aligned}
$$

By the above and (6.1) we obtain

$$
\operatorname{osc}_{B}^{2} f_{a} \leq \frac{C r^{2}}{|c|^{2} \log ^{2} \frac{1}{|c|}} \leq C \int_{B} g(|x|) d x \leq \int_{B} \theta .
$$

Let $0 \in B$. Then $|c| \leq r$. Without loss of generality $c=\left[c_{1}, 0\right]$. Clearly $A=B(0, r) \cap\left\{\left[x_{1}, x_{2}\right]:\left|x_{2}\right| \leq x_{1}\right\} \subset B$. In polar coordinates we have

$$
\begin{aligned}
\int_{B} \theta & \geq C \int_{A} h(|x|) d x=C \int_{-\pi / 4}^{\pi / 4} d \varphi \int_{0}^{r} h(t) t d t \\
& =C \int_{0}^{r}\left(\frac{1}{\log \left(\log \frac{1}{2 t}\right)}\right)^{\prime} d t=C \frac{1}{\log \left(\log \frac{1}{2 r}\right)} .
\end{aligned}
$$

By the above and

$$
\operatorname{Osc}_{B} f_{a} \leq \operatorname{osc}_{B(0,2 r)} f_{a} \leq \frac{2}{\sqrt{\log \left(\log \frac{1}{2 r}\right)}}
$$

we have $\operatorname{osc}_{B}^{2} f_{a} \leq \int_{B} \theta$. Thus we have verified the $R R_{1 / 2}$-condition for $f_{a}$ with weight $\theta$. Hence Theorem 5.1 shows that $f_{a} \in A C_{1 / 2}^{2}$. 
Set

$$
a_{k}=e^{-e^{\pi / 2+2 k \pi}} \quad \text { and } \quad b_{k}=e^{-e^{2 k \pi}} \quad \text { for } k \in \mathbb{N} \text {. }
$$

Clearly

$$
B_{k}=B\left(\left[\frac{a_{k}+b_{k}}{2}, 0\right], \frac{b_{k}-a_{k}}{2}\right), \quad k \in \mathbb{N},
$$

are pairwise disjoint balls. Thus

$$
\begin{aligned}
V_{1}^{2}\left(f_{a}, B(0,1 / 8)\right) & \geq \sum_{k \in \mathbb{N}} \operatorname{osc}_{B_{k}}^{2} f_{a} \geq \sum_{k \in \mathbb{N}}\left|f_{a}\left(a_{k}\right)-f_{a}\left(b_{k}\right)\right|^{2} \\
& =\sum_{k \in \mathbb{N}}\left|\frac{1}{\sqrt{\pi / 2+2 k \pi}}-0\right|^{2}=\infty
\end{aligned}
$$

It follows that $f_{a} \notin B V_{1}^{2}$ and $f_{a} \notin A C_{1}^{2}$.

Acknowledgements. The author would like to thank Professor L. Zajíček for suggesting the problem. The author gratefully acknowledges the many helpful suggestions and valuable comments of Professor J. Malý during the preparation of the paper.

\section{References}

[1] M. Csörnyei, Absolutely continuous functions of Rado, Reichelderfer and Malý, J. Math. Anal. Appl. 252 (2000), 147-166.

[2] H. Federer, Geometric Measure Theory, Springer, 1969, 1996.

[3] S. Hencl and J. Malý, Absolutely continuous functions of several variables and bilipschitz mappings, in preparation.

[4] J. Kauhanen, P. Koskela and J. Malý, On functions with derivatives in a Lorentz space, Manuscripta Math. 100 (1999), 87-101.

[5] J. Malý, Absolutely continuous function of several variables, J. Math. Anal. Appl. 231 (1999), 492-508.

[6] - Sufficient conditions for change of variables in integral, in: Proc. on Analysis and Geometry (Novosibirsk, 1999), Izdat. Ross. Akad. Nauk Sib. Otd. Inst. Mat., Novosibirsk, 2000, 370-386.

[7] T. Rado and P. V. Reichelderfer, Continuous Transformations in Analysis, Springer, 1955.

Department of Mathematical Analysis

Charles University

Sokolovská 83

18600 Praha 8, Czech Republic

E-mail: hencl@karlin.mff.cuni.cz

Received 15 March 2001;

in revised form 7 January 2002 